\title{
SIMO Satellite Communication Systems over Ka Band at Venezuelan Amazonian in Rain Fading Scenarios
}

\author{
Gustavo Guedez ${ }^{1, a}$,Qiang Gao ${ }^{1, b}$ and María Guarirapa ${ }^{1, c}$ \\ ${ }^{1}$ School of Electronic and Information Systems, Beihang University, Beijing,China \\ agguedezcarias@hotmail.com, bgaoqiang@buaa.edu.cn, catenea121@hotmail.com
}

Keywords:Rain Fade, ITU, C/No, Ka Band, SIMO, Capacity, SNR, BER.

\begin{abstract}
The Amazon has the world's largest tropical forest and about the half of all freshwater in the world. The construction of the wire guided communications systems is denied byinternational environment regulation because the Amazon is a worldwide protected area.Satellite communications systems represents the best option to provide communications services to the Amazonian region. However, the quality and availability of the signals in Ka band are seriously degraded by various climate phenomena, especially the rain [1]. The footprint of the Venezuelan Satellite has a large coverage service area over the Amazonian. The rain fade leads to the degradation of the link quality with unacceptable error rate and lower system capacities. In order to overcome this problem, and keep the link properties in normal conditions (clear sky conditions) the diversity concept can be used. The diversity principle is based on the transmission of multiple copies of the same signal. The copies of the signals can be combined at the receiver or be selected the best copy of the signal. The research aim is is modeling the channel attenuation for Ka band and simulate the performance of the Venezuelan Satellite with several receive antennas similar to a Single Input Multiple Output (SIMO) system. The main purpose, is to analyze the system capacity and bit error rate of BER with lower values of Signal to Noise Ratio (SNR) and compare the results with Single Input Single Output (SISO) performance.
\end{abstract}

\section{Introduction}

The Amazon contain the biggest reserve of freshwater and the largest tropical forest in the world. The international regulation protect the Amazonian for that reasonthe satellite technology becomes the best method to provide communications services. However, the rainfalls introduce an important attenuation for Ka band signals [2]. The reduction of the signal level on the channel produces the interruption of the services at high frequencies. Before implementing any mitigation technique, the attenuation level have to be estimated. The climate losses mostly depend of frequency, geographic location and elevation angle [3, 4, 5, 6]. To mitigate the rain effects the diversity concept can be used. Diversity is defined by the transmission of multiple copies from the same signal. The receiver will combine each signal or select the best copy of the signals. The probability thatall the copies has poor quality is very low [7].The Venezuelan Satellite in Ka band can be modeled as a SIMO system, because useone antenna working in a single polarization. The diversity improve thereliability performance of the communication system. The diversity must generate copies of the signal independent to each other, in order to minimize the probability that all the signals face simultaneously bad propagation conditions [7].Normally for satellite communication, the channel can be modelled as the Rice distribution [7, 8]. Rice distribution consist of communication paths which as a strong line of sight (LOS) component combined with several weaker multipath components [7]. The multipath component can be modelled as a Rayleigh distribution [8].The main purpose of the research, is to analyze the system capacity and bit error rate of BER over the rainfall channel and compare the results with the SISO system performance. 


\section{System Model}

\subsection{VENESAT SIMO System}

The Venezuelan satellite can be modeled as a SIMO system. The satellite transmit to several earth station. The earth station has fixed antennas and the satellite is only able to transmit in one polarization as is shown in Fig.1.

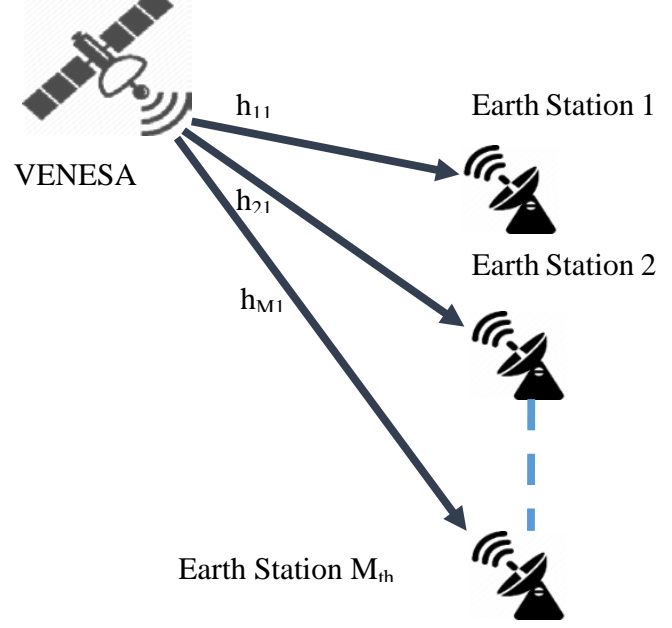

Fig.1 VENESAT transmission to Multiple Earth Stations

The Term $h_{M 1}$ is the channel between the satellite antenna and the $\mathrm{M}_{\mathrm{th}}$ earth station. The received signal at the earth [9].

$$
\left(\begin{array}{c}
\mathrm{y}_{1} \\
\mathrm{y}_{2} \\
\vdots \\
\mathrm{y}_{\mathrm{M}}
\end{array}\right)=\left(\begin{array}{c}
\mathrm{h}_{11} \\
\mathrm{~h}_{21} \\
\vdots \\
\mathrm{h}_{\mathrm{M} 1}
\end{array}\right)(\mathrm{x})+\left(\begin{array}{c}
\mathrm{n}_{1} \\
\mathrm{n}_{2} \\
\vdots \\
\mathrm{n}_{\mathrm{M}}
\end{array}\right)
$$

$Y=H x+n$

The term $\mathrm{y}=\left[\mathrm{y}_{1}, \mathrm{y}_{2}, . ., \mathrm{y}_{\mathrm{M}}\right]^{\mathrm{T}}$ is an $\mathrm{Mx} 1$ vector received signal, $\mathrm{x}$ is the signal transmitted and $\mathrm{n}=$ $\left[\mathrm{n}_{1}, \mathrm{n}_{2}, . ., \mathrm{n}_{\mathrm{M}}\right]^{\mathrm{T}}$ is an Mx1 vector of Gaussian noise with $\mu=0$ and variance $\sigma^{2}$.

\section{Channel Model}

\subsection{Attenuation by rain, other precipitation and clouds}

The rain fade is the dominant and most important factor for frequencies above 10GHz [10].Rain attenuation mainly depends on: frequency, location, polarization and rainfall rate $[1,2,3,5,6]$. The depth of attenuation in $\mathrm{dB}$ for the Venezuelan location can be calculated from:

$\mathrm{L}_{\text {rain }}=$ rain loss $(\mathrm{dB})$

$\gamma_{\mathrm{r}}$ specific attenuation $(\mathrm{dB} / \mathrm{Km})$

Drain= path length $(\mathrm{Km})$

$\mathrm{h}_{\mathrm{r}}=5$ rain height $(\mathrm{Km})$

$\theta=$ elevation angle $\left({ }^{\circ}\right)$.

ho= sea level height $(\mathrm{Km})$. 
TABLE I. RAINFALL RATE

\begin{tabular}{|c|c|c|c|c|c|}
\hline \multirow{2}{*}{$\begin{array}{c}\text { Percentage of time R } \\
\text { exceeded }\end{array}$} & \multicolumn{5}{|c|}{ Zone } \\
\cline { 2 - 6 } & $\mathbf{L}$ & $\mathbf{M}$ & $\mathbf{N}$ & $\mathbf{P}$ & $\mathbf{Q}$ \\
\hline 1 & 2 & 4 & 5 & 12 & 24 \\
\hline 0,3 & 7 & 11 & 15 & 34 & 49 \\
\hline 0,1 & 15 & 22 & 35 & 65 & 72 \\
\hline 0,03 & 33 & 40 & 65 & 105 & 96 \\
\hline 0,01 & 60 & 63 & 95 & 145 & 115 \\
\hline 0,003 & 105 & 95 & 140 & 200 & 142 \\
\hline 0,001 & 150 & 120 & 180 & 250 & 170 \\
\hline
\end{tabular}

The rainfall rate "R", for a $0.1 \%$ of excess rain, mean a $99.9 \%$ of service availability. Venezuela is located in zone $\mathrm{N}$ [1].For a determinate polarization in $\mathrm{Ka}$ band $(20 \mathrm{GHz})$, the coefficients $\mathrm{k}$ and $\alpha$ are chosen according to the table 2.

TABLE II. RAINFALL COEFFICIENT

\begin{tabular}{|c|c|c|c|c|}
\hline $\begin{array}{c}\text { Frequency } \\
\text { (GHz) }\end{array}$ & kh & $\mathbf{k v}$ & $\boldsymbol{\alpha h}$ & $\boldsymbol{\alpha v}$ \\
\hline 15 & 0,03689 & 0,3362 & 1,1549 & 1,1275 \\
\hline 20 & 0,07504 & 0,06898 & 1,0995 & 1,0663 \\
\hline 25 & 0,1237 & 0,1125 & 1,0604 & 1,0308 \\
\hline
\end{tabular}

For the uplink signals, the thermal noise is not relevant because the antennas of the satellite are pointing to hot spot on earth. In downlink unfortunately, the rainfall can introduce a significant quantity of thermal noise [10]. $\mathrm{T}_{\mathrm{a}}$ is the apparent absorber temperature between $270^{\circ} \mathrm{K}$ and $290{ }^{\circ} \mathrm{K}$.

$$
\begin{aligned}
& A_{t}=10^{\frac{A}{10}}(3) \\
& T_{\text {rain }}=T_{a}\left(1-\frac{1}{A_{t}}\right)(4) \\
& T_{\text {sky }}=T_{\text {cs }}+T_{\text {rain }}(5)
\end{aligned}
$$

\subsection{Free Space Loss Model}

The free space SIMO model is based on the line of sight (LOS) component of the channel. The channel model can be represented $[8,9]$.

$$
\boldsymbol{H}_{i j}=\boldsymbol{\alpha}_{i j} * \boldsymbol{e}^{\left(-j k_{0} f_{c} \boldsymbol{r}_{i}\right)}(6)
$$

Wherer $r_{i}$ is the distance between the satellite and the $i_{\text {th }}$ earth station antenna, $f_{c}$ is the carrier frequency, $\mathrm{k}_{0}=(2 * \pi / \mathrm{co})$ and co is the speed of light. The term $\varphi$ is assumed equal to zero (phase)and $\alpha$ is approximated to a constant. The propagation path attenuation can be defined [8].

$$
\alpha_{i j}=\frac{1}{2 k_{0} f_{c} r_{i}} * e^{(-j \varphi)}
$$




\subsection{VENESAT Analytical Channel Model}

The channel model follow a Rice distribution which has the LOS component and non LOS component (multipath component) [11].The multipath effects is not significant compare with the LOS component. The channel is defined by the carrier frequency and the geostationary distance.The capacity for SIMO can be calculated from Eq. 8. [7].

$$
C=\log _{2}(1+\mathrm{SNR} *\|h\|)
$$

The term $\|$. $\|$ is the norm of the matrix and SNR is the signal to noise ratio received on the earth station antennas. The SNR in decibel is compute from Eq. 9. [10].

$S N R=\mathrm{SNR}_{\text {Clear sky }}-$ Attenuation $_{\text {Rainfall }}$

The probability of obtain an error due the noise [10]

$P_{e}=\frac{1}{2} \operatorname{erfc}\left(\sqrt[2]{\frac{1}{2} S N R}\right)$

\section{Simulation Results}

\subsection{Attenuation by rain, other precipitation and clouds}

The simulation follows the ITU recommendations procedure [2]. The input data related to a ground station are: latitude, height over the sea level and antenna elevation. The rain effects are calculated for a statistic of one year with different availability of services: 99\%, 99.7\%, 99.9\%, 99.97\%, 99.99\%, 99.997\% and 99.999\%.

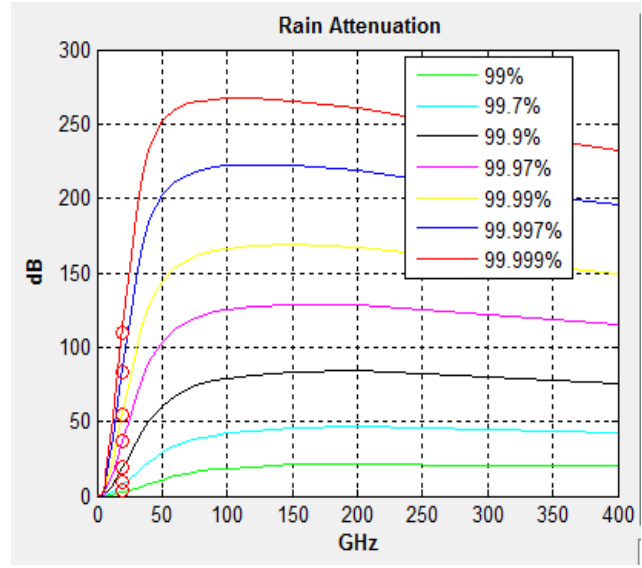

Fig. 2 Simulation of attenuation by rain, other precipitation and clouds.

\subsection{Ergodic Capacity and Bit Error Rate}

The ergodic capacity and bit error rate (BER) estimation is based in a SIMO system with four receive antennas.The BER assume signals transmitted over a Rice channel with BPSK modulation. The channel follow the Eq.6. The multipath effects are considered equal to zero, due the high elevation angles at the Venezuelan Amazon region. The simulation result are shown in Fig. 3. 


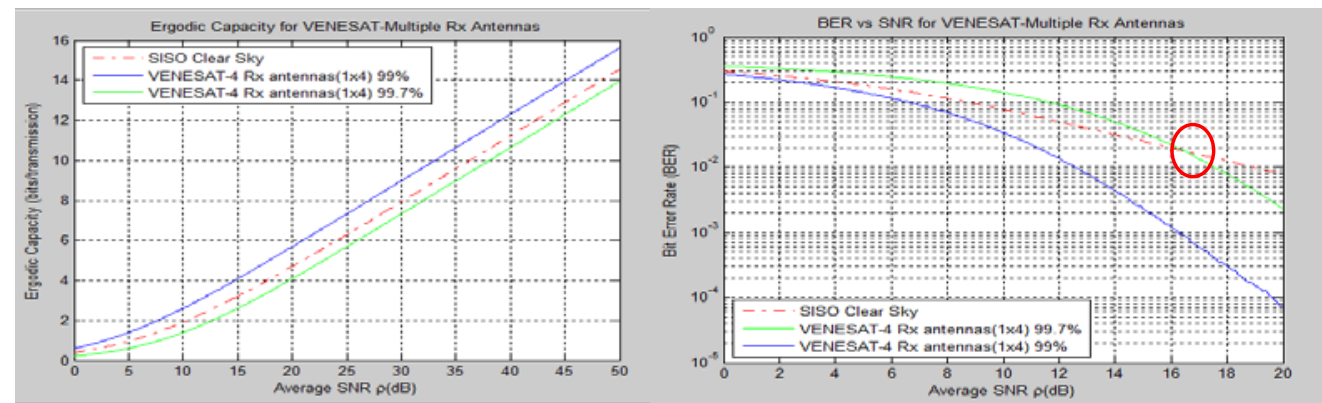

Fig. 3 Simulation of Ergodic Capacity and BER.

For $99 \%$ of services availability, the capacity has a better performance than the SISO system without presence of rain (clear sky conditions). In order to guarantee $99.7 \%$ of services availability SIMO system is not able to maintain the clear sky conditions for low levels of SNR. However, for $99.7 \%$ of availability, the probability of error decrease significantly for high levels of SNR.

\section{Conclusion}

Satellites communication signals working at Ka band frequencies, are degradeddue rainfall. Scintillation and multipath effects can be considered equal to zero because the Venezuelan earth station antennas are close to equator without obstacle for the field of view. However, attenuation by rain, other precipitation and clouds is extremely high for Ka bands. For a service availability of 99\% and $99.7 \%$ the probability of error is improved considerably, especially at high levels of SNR.

\section{References}

[1] ITU R P.618-11, "Propagation data and prediction methods required for the design of Earth-space telecommunication systems" (2013).

[2] ITU R P.839-3, "Rain height model for prediction methods" (2001).

[3] ITU-R P.676-10, "Attenuation by atmospheric gases" (2013).

[4] ITU-R P.835-5, "Reference standard atmospheres" (2012).

[5] ITU-R P.1510, "Annual Mean Surface Temperature" (2001).

[6] ITU-R P.453-8 "The radio refractive index: its formula and refractivity data" (2001).

[7] T.Brown, E. De Carvalho, P. Kyritsi “Practical Guide to the MIMO Radio Channel” New York: WILEY 2012

[8] M.Simon, M.Alouini “Digital Communication over Fading Channels” New York: WILEY 2012.

[9] Ramoni Adeogun, "Capacity and Error Rate Analysis of MIMO Satellite Communication Systems in Fading Scenarios,” International Journal of Electrical and Computer Engineering (IJECE), August 2014, vol. 4 pp. 612-622.

[10] D. Rodis, “Satellite Communications,” New York: McGraw-Hill 2006.

[11] Loo, C. “A statistical Model for a Land Mobile Satellite Link,” IEEE transacction of vehicular Technology, 1998 vol. 34 pp. 122-127. 